\title{
Modelo de Costos Completo para Empresas de Carga Marítima: Caso de Aplicación
}

\author{
Norma Pontet Ubal \\ Doutorado de la Universidad ORT Uruguay \\ Bvar. España 2633, Montevideo \\ E-mail: norma.pontet@ort.edu.uy
}

\section{RESUMO}

El presente trabajo es una exposición de resultados del estudio realizado para una empresa de transporte marítimo en el período 2007/2008, cuyo objetivo principal ha sido diseñar un sistema racional de costes en una empresa naviera, que proporcione la información necesaria para una adecuada asignación de costes. La elección metodológica ha sido fundamentalmente cualitativa. Todas las fases del proyecto se articulan en torno a un doble objetivo: por una parte, el análisis desde una perspectiva teórica y aplicada de los factores explicativos y los efectos asociados a los intercambios entre los países de escala de navegación, pertenecientes al propio caso analizado y sus respectivas áreas de influencia. Se ha trabajado con costes operativos, financieros y con la adecuada exposición y tratamiento de la capacidad ociosa en un estándar de eficiencia previamente definido por la organización. Al efectuar este análisis se observó que las empresas del sector tienen una gran proporción de costes fijos en su estructura de costes, algunos de los cuales son fijos respecto al viaje y otros son fijos totales al nivel de la empresa, por lo que el inadecuado tratamiento de los mismos podría derivar en decisiones erróneas.

Palabras clave: Capacidad ociosa. Eficiencia. Costos fijos.

Full cost model for sea freight companies: Case Application

\section{ABSTRACT}

This paper is an exposition of results of the study for a shipping company in the period 2007/2008. They have to present the main lines of work, whose main objective was to design a rational system of costs in a shipping company that provides the information necessary for the proper allocation of costs. The choice has been primarily qualitative methodology. All phases of the project is structured around two objectives: firstly, the analysis from a theoretical and applied the explanatory factors and the effects associated with trade between the countries of scale navigation, belonging to the very case in question and their respective areas of influence. We have worked in operational costs, financial and with adequate exposure and treatment of unused capacity on a standard of efficiency previously defined by the organization. Companies from this sector have a great proportion of fixed costs, some of which are fixed in relation to travel 
and others are fixed at the level of total business, so that an inadequate treatment of these costs could lead to wrong decisions.

Key Words: Capacity. Efficiency. Fixed costs.

\section{INTRDUCCIÓN}

Para diversos autores la contabilidad de costes en empresas de servicios se transformó en una necesidad estratégica en las últimas décadas. Esto se tornó más evidente cuando las presiones competitivas mundiales modificaron la naturaleza de la economía y muchas empresas debieron cambiar la forma en la que operaban sus negocios (BACIC, 2006; PUNAKIVI; HINKKA, 2006; LIEB; RANDALL, 1999; CRESPO et al., 2005; DE CORLA et al., 1997).

La empresa de transporte de carga marítima, como potencial prestadora de un servicio, hace uso de esta contabilidad de costes, que a su vez se ha transformado en apoyo fundamental para afrontar la realidad y tomar decisiones vinculadas al corto, mediano y largo plazo (GINER et al., 2008). En tal sentido, destacan Giner y Ripoll (2007) que la misma le aporta una herramienta fundamental para la toma de decisiones exitosas, y le otorga la capacidad de discernir qué "coste" es el más útil para la decisión a tomar y no la condición de "absoluto" de los datos que maneja.

El presente trabajo es una exposición de resultados del estudio realizado para una empresa de transporte marítimo en el período 2007-2008. Se exponen las principales líneas del trabajo, encontrándose a disposición de los interesados el estudio completo, cuyo objetivo principal ha sido diseñar un sistema racional de costes en una empresa naviera, que proporcione la información necesaria para una adecuada asignación de costes.

Como resultado de la aplicación del sistema, se pretende que la información obtenida permita:

- Efectuar estudios económicos de rentabilidad de servicios potenciales o nuevos proyectos (líneas de servicio).

- Evaluar la operación y/o productividad de líneas de servicio específicas. 
- Conocer el margen de la cartera de líneas de la empresa, así como también el margen total de la misma.

- Determinar las ineficiencias existentes en la operativa de las diferentes líneas.

- Fomentar una cultura de mejora continúa de la gestión organizativa implantando sistemas de evaluación y control.

\section{BASES CONCPTUALES APLICADAS}

Siguiendo a AECA (2006), se aplicó un modelo de costeo completo, para el que se determinaron los objetivos de costes entendidos como "aquello para lo cual se desea una medición separada de costes" (HORNGREN et al.,1996). En la empresa bajo análisis, dependiendo del ciclo de los viajes, la clase de buques y el tipo de operación que se realice, puede convenir definir el objeto de costes por líneas separadas, por buques o bien por periodos de tiempo, en función de las necesidades de información, ya que los objetivos no se eligen por sí mismos, sino con la finalidad de tener un apoyo en la toma de decisiones. El presente sistema define un objetivo de costes final, así como también objetivos de costes intermedios, siendo estos el instrumento a través del cual se obtiene el coste del objetivo final. El objetivo final de costes será una línea con su frecuencia regular, es decir el viaje redondo de cada uno de los buques que completan la línea y su frecuencia.

En tal sentido, la elección de este objetivo final de costes se asocia a la base de líneas regulares, para las que se pre-establecen rutas, distancias, puertos de escala y velocidad, entre otros, de modo que cada una de dichas líneas se puede considerar como un servicio independiente. Esto implica que para evaluar la eficiencia en la prestación del servicio, se deberá analizar la eficiencia de la operación de dichas líneas, y por ende, se requiere conocer el coste de cada una (GINER et al., 2007).

Se han definido objetivos intermedios principales que se relacionan con la función básica de prestación del servicio de transporte de carga marítimo. Asimismo, se los puede sub-clasificar en los siguientes tres tipos:

1. Directos: Transfieren los costes de los recursos consumidos directamente a una sola línea de servicios del total de líneas que maneja la empresa. Para la 
empresa de referencia, se define como objetivo de costes intermedio principal directo, a las operaciones por movimiento de la carga operada por cada una de las líneas que explota la empresa. Para costear dicho objetivo intermedio, se acumularán todos los gastos originados por las operaciones por movimiento de la carga de cada línea, en el centro de costes denominado "Carga línea x".

2. No directos: Transfieren los costes de los recursos consumidos indirectamente a distintas líneas de servicios de la cartera de líneas, siguiendo algún método de distribución o adjudicación de costes. En las empresas de transporte de carga marítimo se define como objetivos de costes intermedios principales no directos a las operaciones por movimiento de cada buque (round trip), que conforma la flota de la empresa. Para costear dicho objetivo intermedio, se acumularán todos los repartos originados por las operaciones por movimiento de cada buque, en el centro de costes denominado "Buque a", para luego ser adjudicados a la cartera de líneas, en función de los días que cada línea consume o utiliza un buque.

3. De estructura (Ociosidad): Transfieren los costes de los recursos fijos no utilizados en la prestación del servicio, y que por tanto, no deben imputarse a la cartera de líneas de la empresa, sino que deben ser considerados como costes del periodo, formando parte de la estructura general de la empresa y afectando únicamente al resultado de explotación de la misma. En las empresas de transporte marítimo de contenedores se definen como objetivos de coste intermedio de estructura a la capacidad no utilizada, o sea la capacidad ociosa de producción. Para costear dicho objetivo se acumularán todos los costes asociados a capacidad ociosa de producción en el centro de costes denominado "Estructura".

Por otra parte los centros de costes auxiliares son receptores de costes que se asignan, posteriormente, en función de las unidades de obra o actividad entre los objetivos de costes principales (directos, no directos y de estructura) e, inclusive, a otros objetivos de costes auxiliares, en caso de existir. Su finalidad es servir de apoyo a la función básica del servicio de transporte de carga marítimo. Sus consumos repercuten de forma indirecta en la cartera de líneas a través de los objetivos de costes principales. 
Se resumen en el Cuadro 1 los objetivos de costes antes descriptos.

\begin{tabular}{|c|c|c|}
\hline Objetivo de costes & Clasificación & Descripción \\
\hline Final & $\begin{array}{l}\text { Servicio } \\
\text { prestado }\end{array}$ & $\begin{array}{c}\text { Viaje redondo de una línea con su frecuencia } \\
\text { regular }\end{array}$ \\
\hline \multirow[t]{4}{*}{ Intermedio } & \multirow[t]{3}{*}{ Principales } & $\begin{array}{c}\text { Directos: operaciones de movimiento de la carga } \\
\text { operada por cada línea. }\end{array}$ \\
\hline & & $\begin{array}{c}\text { No Directos: operaciones de movimiento de cada } \\
\text { uno de los buques que conforman la flota de la } \\
\text { empresa. }\end{array}$ \\
\hline & & $\begin{array}{l}\text { De Estructura: capacidad no utilizada, capacidad } \\
\text { ociosa de producción. }\end{array}$ \\
\hline & Auxiliares & $\begin{array}{l}\text { Son receptores de costes que se distribuyen, en } \\
\text { función de diversos criterios a los objetivos } \\
\text { principales. Sirven de apoyo a los principales, por } \\
\text { ejemplo operaciones de seguridad. }\end{array}$ \\
\hline
\end{tabular}

Cuadro 1 - Objetivos de Costes

\section{ESTRATEGIA DE INVESTIGACIÓN Y METODOLOGÍA}

Para llevar a cabo una investigación empírica, existen diferentes métodos de investigación alternativos, la elección de uno u otro dependerá, entre otras variables, de las características y de la naturaleza de la investigación. A priori, ninguna es mejor que otra, ya que todas tienen ventajas y limitaciones, por ello algunos expertos en metodología de investigación abogan por una combinación de cuantitativa y cualitativa con el objeto de incrementar la fiabilidad de los estudios realizados (BREWER; HUNTER, 1989).

En ese caso, la elección ha sido de una metodología fundamentalmente cualitativa, dado que los problemas y cuestiones planteados en los objetivos son aspectos dinámicos difíciles de estudiar mediante metodología estrictamente cuantitativa. Todas las fases del proyecto se articulan en torno a un doble objetivo: por una parte, el análisis desde una perspectiva teórica y aplicada de los factores explicativos y los efectos asociados a los intercambios entre los países de escala de navegación, pertenecientes al propio caso analizado y sus respectivas áreas de influencia. Por otra, en el estudio se han tomado los costes del período 2007-2008. Se ha obtenido información de la contabilidad financiera realizada de acuerdo a 
NICs/NIIFs, de la elaboración de su presupuesto para el mismo período y del estándar con un nivel de eficiencia previamente definido por la organización.

Estos costes representan el valor de los recursos consumidos por la empresa en la prestación del servicio de transporte marítimo de contenedores en ese período. Para este caso, los recursos identificados como relevantes y su análisis de sensibilidad, se exponen en el Cuadro 2.

\begin{tabular}{|c|c|c|c|}
\hline \multirow[b]{3}{*}{ Recurso o factor } & \multicolumn{3}{|c|}{ Sensibilidad de los factores } \\
\hline & \multirow[t]{2}{*}{ Variables } & \multicolumn{2}{|c|}{ Fijos } \\
\hline & & Capacidad & Operativos \\
\hline Combustible & & & $\square$ \\
\hline Água & & & $\square$ \\
\hline Avituallamiento & & & $\square$ \\
\hline Tripulación & & & $\square$ \\
\hline Personal de la empresa armadora en tierra & & $\square$ & \\
\hline Servicios portuários & & & $\square$ \\
\hline Derecho de paso por canales & & & $\square$ \\
\hline Charteo de buquês & & & $\square$ \\
\hline Manejo de contenedores & $\square$ & & \\
\hline $\begin{array}{c}\text { Administración flota de contenedores (excepto } \\
\text { alquiler) }\end{array}$ & & $\square$ & \\
\hline Alquiler de contenedores & & & $\square$ \\
\hline Comunicaciones en viaje & & & $\square$ \\
\hline Reparación y mantenimiento de buquês & & & $\square$ \\
\hline Reparación y mantenimiento de contenedores & & $\square$ & \\
\hline Seguros de la carga & $\square$ & & \\
\hline Seguros del buquê & & & $\square$ \\
\hline $\begin{array}{c}\text { Bienes y servicios contratados } x \text { empresa } \\
\text { armadora en tierra }\end{array}$ & & $\square$ & \\
\hline Servicios de agencia & $\square$ & & \\
\hline Servicios de vigilância & & $\square$ & \\
\hline Recursos financieros & & $\square$ & \\
\hline $\begin{array}{l}\text { Pérdida de potencialidad o depreciación de flota } \\
\text { de buques }\end{array}$ & & & $\square$ \\
\hline $\begin{array}{l}\text { Pérdida de potencialidad o depreciación de flota } \\
\text { de contenedores }\end{array}$ & & $\square$ & \\
\hline Capacidad ociosa $\left({ }^{*}\right)$ & & & \\
\hline
\end{tabular}

Cuadro 2 - Análisis de sensibilidad de los Factores

(*) La capacidad ociosa es la porción de los factores de costes fijos de capacidad y operativos no utilizados en el proceso productivo. 
El valor de los recursos consumidos es acumulado en los centros de costes del sistema, configurando así la distribución primaria de los costes a los objetivos de costes principales y auxiliares. Para efectuar tal distribución se deberán clasificar los factores de costes de acuerdo a su direccionalidad, es decir, en directos e indirectos a cada uno de los objetivos de costes intermedios. El criterio seguido para ello, se desprende de la propia definición de direccionalidad, por tanto, los factores de costes que se encuentran clara e inequívocamente vinculados al objetivo de costes operaciones de carga por línea de servicios. Los factores de costes directos al objetivo de costes buques son todos aquellos relacionados con el movimiento del buque por realizar el viaje redondo.

Los factores de costes directos al objetivo de costes de estructura son los factores fijos tanto estructurales como operativos no utilizados en la producción. La distribución secundaria, tiene como finalidad asignar los costes de los objetivos de costes auxiliares a los objetivos de costes principales, en función del uso que de los primeros hicieron los segundos. Al término de este proceso los objetivos auxiliares quedan anulados. En el Cuadro 3 se expone el resultado del análisis de direccionalidad de los costes en relación con los objetivos de costes: 


\begin{tabular}{|c|c|c|c|c|c|c|c|c|}
\hline \multirow[b]{4}{*}{ Recurso o factor } & \multicolumn{8}{|c|}{ Direccionalidad de los factores } \\
\hline & \multirow{2}{*}{\multicolumn{2}{|c|}{$\begin{array}{l}\text { Op. De } \\
\text { Carga }\end{array}$}} & \multirow{2}{*}{\multicolumn{2}{|c|}{$\begin{array}{l}\text { Op. de } \\
\text { Buques }\end{array}$}} & \multirow{2}{*}{\multicolumn{2}{|c|}{ Estructura }} & \multirow{2}{*}{\multicolumn{2}{|c|}{$\begin{array}{c}\text { Op. de } \\
\text { Vigilancia }\end{array}$}} \\
\hline & & & & & & & & \\
\hline & Dir. & Indir. & Dir. & Indir. & Dir. & Indir. & Dir. & Indir. \\
\hline Combustible & & & $\square$ & & & & & \\
\hline Agua & & & $\square$ & & & & & \\
\hline Avituallamiento & & & $\square$ & & & & & \\
\hline Tripulación & & & $\square$ & & & & & \\
\hline $\begin{array}{l}\text { Recursos humanos de la } \\
\text { empresa armadora en tierra }\end{array}$ & & $\square$ & & $\square$ & & & & \\
\hline Servicios portuarios & & & $\square$ & & & & & \\
\hline Derecho de paso por canales & & & $\square$ & & & & & \\
\hline Charteo de buques & & & $\square$ & & & & & \\
\hline Manejo de contenedores & $\square$ & & & & & & & \\
\hline $\begin{array}{c}\text { Administración flota de } \\
\text { contenedores (excepto } \\
\text { alquiler) }\end{array}$ & & $\square$ & & $\square$ & & & & \\
\hline Alquiler de contenedores & & $\square$ & & $\square$ & & & & \\
\hline Comunicaciones en viaje & & & $\square$ & & & & & \\
\hline $\begin{array}{c}\text { Reparación y mantenimiento } \\
\text { de buques }\end{array}$ & & & $\square$ & & & & & \\
\hline $\begin{array}{l}\text { Reparación y mantenimiento } \\
\text { de contenedores }\end{array}$ & & & $\square$ & & & & & \\
\hline Seguros de la carga & $\square$ & & & & & & & \\
\hline Seguros del buque & & & $\square$ & & & & & \\
\hline $\begin{array}{l}\text { Bienes consumidos y } \\
\text { servicios contratados por la } \\
\text { empresa armadora en tierra }\end{array}$ & & $\square$ & & $\square$ & & & & \\
\hline Servicios de agencia & $\square$ & & & & & & & \\
\hline Servicios de vigilancia & & $\square$ & & $\square$ & & $\square$ & $\square$ & \\
\hline Recursos financieros & & $\square$ & & $\square$ & & & & \\
\hline $\begin{array}{c}\text { Pérdida de potencialidad o } \\
\text { depreciación de flota de } \\
\text { buques }\end{array}$ & & & $\square$ & & & & & \\
\hline $\begin{array}{l}\text { Pérdida de potencialidad o } \\
\text { depreciación de flota de } \\
\text { contenedores }\end{array}$ & & $\square$ & & $\square$ & & & & \\
\hline Capacidad ociosa $\left(^{*}\right)$ & & & & & $\square$ & & & \\
\hline
\end{tabular}

Cuadro 3 - Análisis de Direccionalidad de los Factores

Los costes de los objetivos de costes principales directos son afectados a la cartera de líneas de forma directa, asimismo, los objetivos de costes principales no directos en función de los consumos que cada una de las líneas absorban de estos centros mediante los criterios de adjudicación. Esta asignación de costes a la cartera de 
líneas, permite obtener el coste por línea. Adicionalmente, si se toma el ingreso obtenido por línea, se puede calcular la rentabilidad por línea, y luego, se llega a la rentabilidad de la empresa y el resultado de explotación.

En resumen, a partir de AECA (2006) se ha propuesto el esquema general del sistema de contabilidad de costes desarrollado en el siguiente esquema:

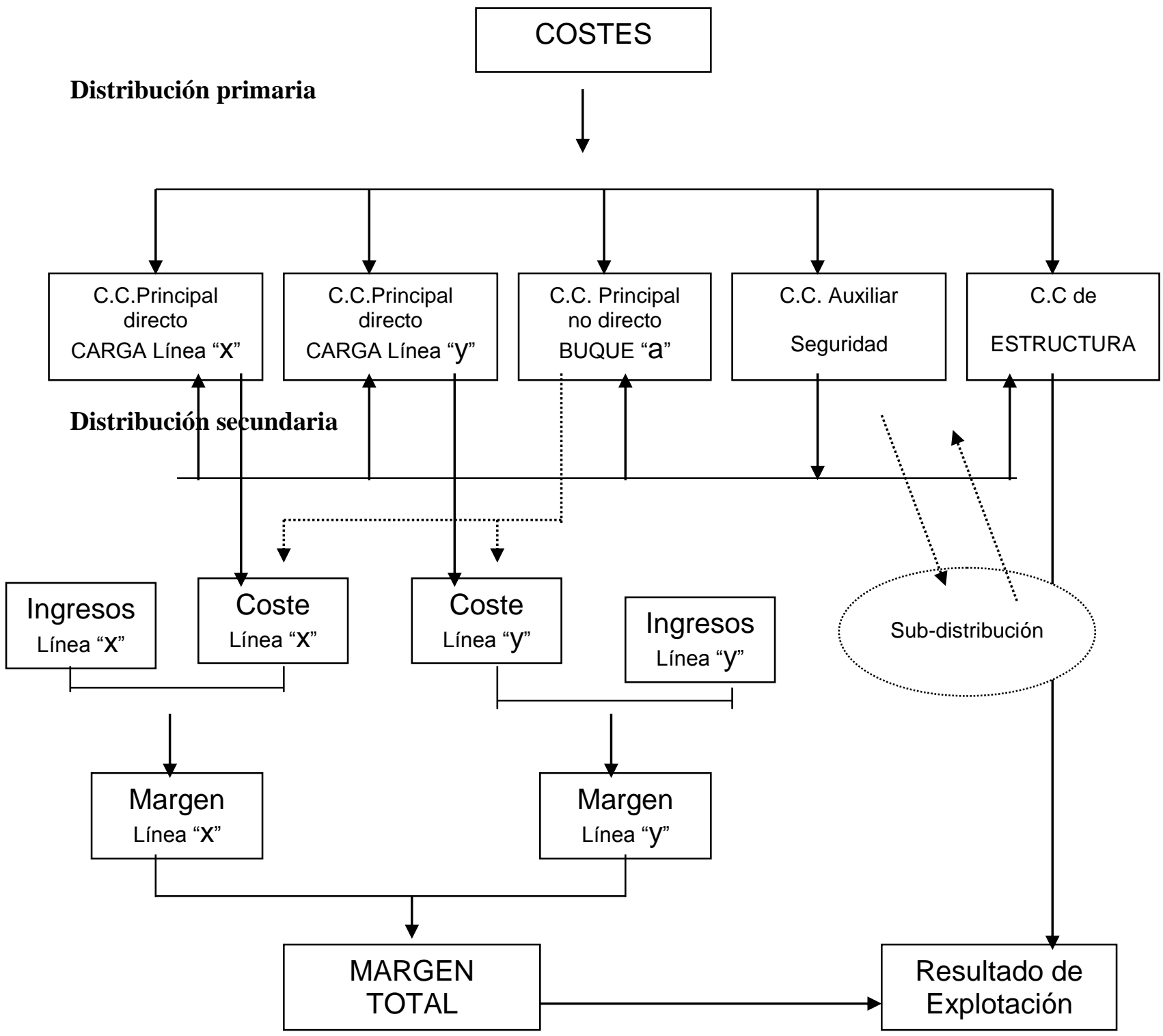

Figura 1 - Distribución de Costes 
Obtenidos los costes correspondientes a las líneas de negocio, comparándolos con los ingresos devengados por cada una de las líneas, el modelo permite determinar el margen de cada una de las líneas, y por adición de los márgenes el margen total. Así, el resultado de explotación se obtiene deduciendo del margen total, derivado de la actividad operativa de la empresa de transporte marítimo, los costes de los centros de coste de estructura o de capacidad ociosa. Conjuntamente, el objetivo final del modelo de costes desarrollado es la adecuada asignación de costes a centros de responsabilidad para determinar la rentabilidad por línea de servicio de transporte marítimo.

El modelo de costes descrito es un instrumento que permite valorar y explicar las diversas transacciones que se efectúan en una empresa de transporte marítimo de contenedores. El modelo permite identificar y determinar los costes directos, no directos, y auxiliares, así como los costes de estructura o capacidad ociosa en las líneas de servicios analizadas.

\section{RESULTADOS DE LA APLICACIÓN DEL SISTEMA DE COSTEO}

Tal como desarrolla Barlett (1986), para establecer una línea de servicios regular se debe investigar y analizar el movimiento de la propia carga, evaluar cuál es el buque más conveniente en tipo y tamaño, evaluar los puertos de escala y la frecuencia del servicio.

En este trabajo se establecieron 3 líneas a partir de datos suministrados por la organización en la que se aplicó. Por otra parte, los datos de las distancias entre puertos y el tiempo que es necesario para recorrerlas de acuerdo a la velocidad a la que la empresa navegará, fueron obtenidos por medio de un sistema de cálculo de la empresa y comparados con los que se encuentran disponibles en algunos sitios web ${ }^{1}$.

\footnotetext{
${ }^{1}$ Se puede acceder a www.distances.com
} 


\section{Línea 1}

\begin{tabular}{|c|c|c|c|c|}
\hline Tramos & $\begin{array}{l}\text { Puerto } \\
\text { Origen }\end{array}$ & Puerto Destino & $\begin{array}{c}\text { Distancia } \\
\text { (millas) }\end{array}$ & $\begin{array}{c}\text { Tiempo (días) } \\
\text { Velocidad } 19 \text { nudos }\end{array}$ \\
\hline \multicolumn{5}{|c|}{ Este-Oeste } \\
\hline Tramo 1 & Rotterdam & Le Havre & 247 & 0,6 \\
\hline Tramo 2 & Le Havre & Baltimore & 3.196 & 7,0 \\
\hline Tramo 3 & Baltimore & New York & 410 & 1,0 \\
\hline Tramo 4 & New York & Norfolk & 172 & 0,38 \\
\hline \multicolumn{5}{|l|}{ Oeste-Este } \\
\hline Tramo 1 & Norfolk & Bremerhaven & 3.747 & 8,2 \\
\hline Tramo 2 & Bremerhaven & Felixstowe & 311 & 0,8 \\
\hline \multirow[t]{3}{*}{ Tramo 3} & Felixstowe & Rotterdam & 121 & 0,3 \\
\hline & & Total días en el mar & 8.204 & 18 \\
\hline & $\begin{array}{l}\text { Total puertos } \\
=7\end{array}$ & $\begin{array}{l}\text { Días prom. en puerto }= \\
1,35\end{array}$ & & 10 \\
\hline \multicolumn{2}{|c|}{ Total Días Round Trip } & & & 28 \\
\hline
\end{tabular}

Cuadro 4 - Línea 1

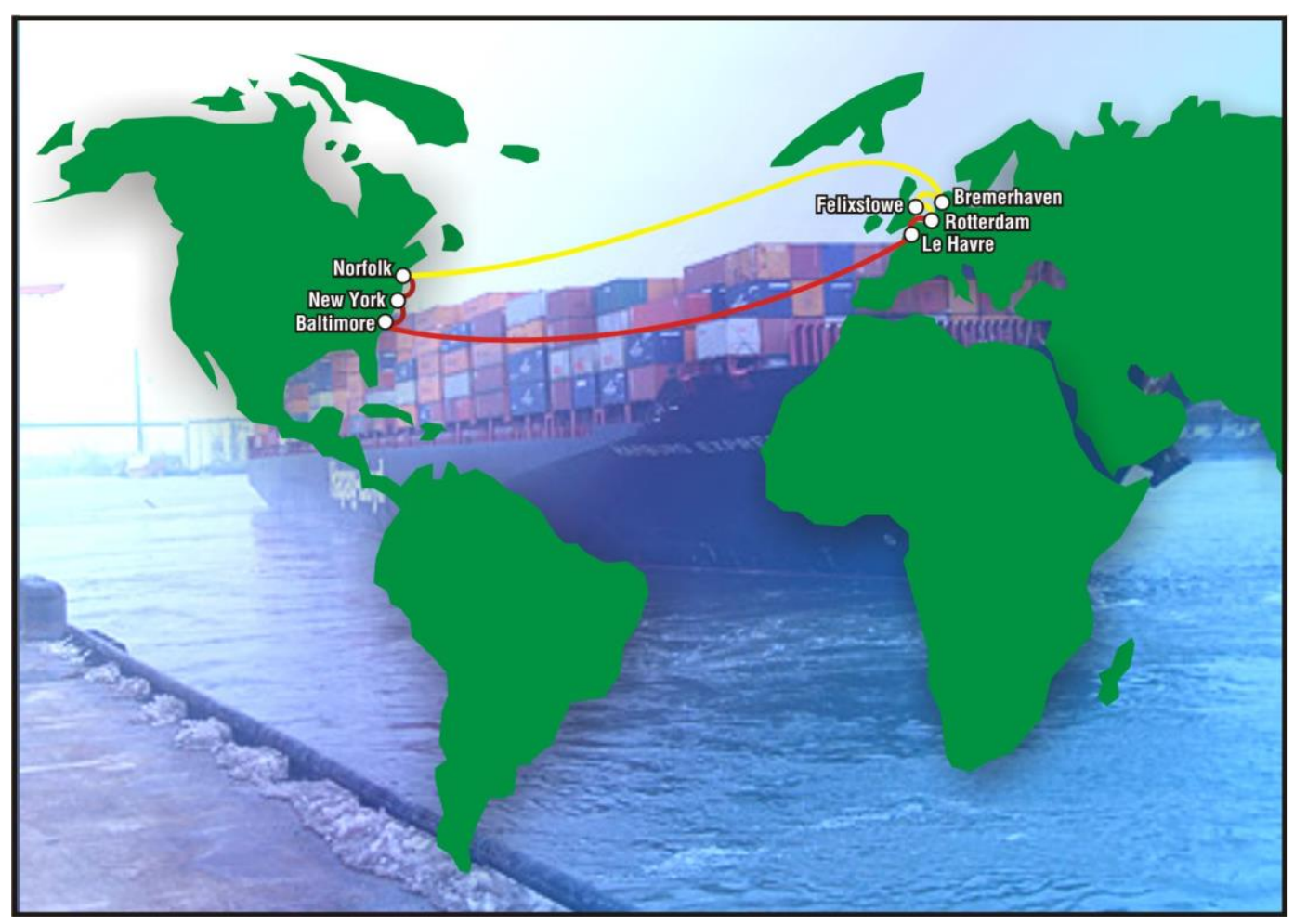

Figura 2 - Ruta Línea 1 


\section{Línea 2}

\begin{tabular}{|c|c|c|c|c|}
\hline Tramos & Puerto Origen & Puerto Destino & $\begin{array}{c}\text { Distancia } \\
\text { (millas) }\end{array}$ & $\begin{array}{c}\text { Tiempo (días) } \\
\text { Velocidad } 20 \\
\text { nudos }\end{array}$ \\
\hline \multicolumn{5}{|l|}{ Sur-Norte } \\
\hline Tramo 1 & Montevideo & Puerto Cabello & 4.501 & 9,3 \\
\hline Tramo 2 & Puerto Cabello & Cartagena & 558 & 1,1 \\
\hline Tramo 3 & Cartagena & Houston & 1.626 & 3,3 \\
\hline Tramo 4 & Houston & New Orleans & 433 & 0,9 \\
\hline Tramo 5 & New Orleans & Miami & 762 & 1,6 \\
\hline \multicolumn{5}{|l|}{ Norte-Sur } \\
\hline Tramo 1 & Miami & Puerto Cabello & 1.235 & 2,46 \\
\hline Tramo 2 & Puerto Cabello & Salvador & 2.814 & 5,8 \\
\hline \multirow[t]{4}{*}{ Tramo 3} & Salvador & Vitoria & 480 & 1,0 \\
\hline & Vitoria & Montevideo & 1.382 & 2,6 \\
\hline & & & 13.791 & 28 \\
\hline & Total puertos $=9$ & $\begin{array}{c}\text { Días prom. en } \\
\text { puerto }=1,35\end{array}$ & & 12 \\
\hline \multicolumn{2}{|c|}{ Total Días Round Trip } & & & 40 \\
\hline
\end{tabular}

Cuadro 5 - Línea 2

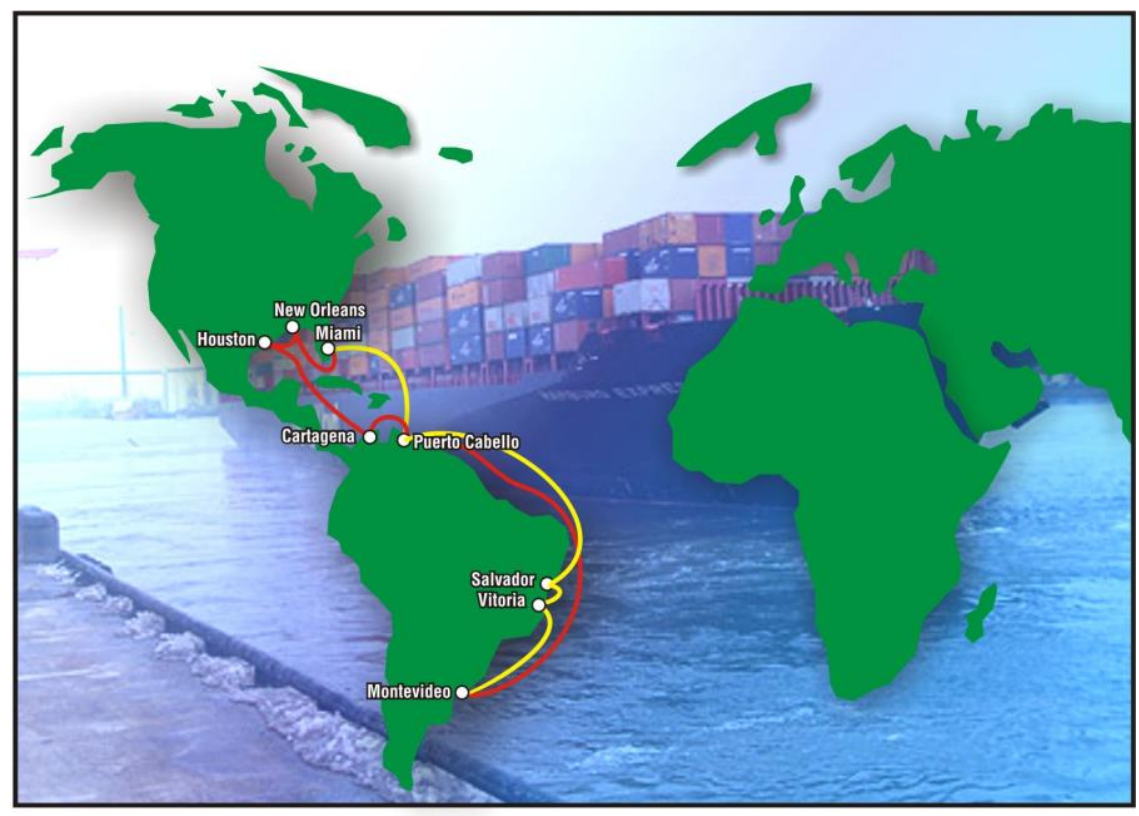

Figura 3 - Ruta Línea 2 


\section{Línea 3}

\begin{tabular}{|c|c|c|c|c|}
\hline Tramos & Puerto Origen & Puerto Destino & $\begin{array}{c}\text { Distancia } \\
\text { (millas) }\end{array}$ & $\begin{array}{c}\text { Tiempo (días) } \\
\text { Velocidad } 20 \\
\text { nudos }\end{array}$ \\
\hline \multicolumn{5}{|l|}{ Sur-Norte } \\
\hline Tramo 1 & Montevideo & Río Grande & 332 & 0,8 \\
\hline Tramo 2 & Río Grande & Paranagua & 496 & 1,3 \\
\hline Tramo 3 & Paranagua & Santos & 155 & 1,0 \\
\hline Tramo 4 & Santos & Río de Janeiro & 210 & 0,7 \\
\hline Tramo 5 & Río de Janeiro & Barcelona & 5.053 & 12,0 \\
\hline Tramo 6 & Barcelona & Valencia & 956 & 0,3 \\
\hline \multicolumn{5}{|l|}{ Norte-Sur } \\
\hline Tramo 1 & Valencia & Dakar & 1.885 & 3,9 \\
\hline Tramo 2 & Dakar & Pointe Noire & 2.228 & 4,6 \\
\hline Tramo 3 & Pointe Noire & Río de Janeiro & 3.375 & 7,5 \\
\hline Tramo 4 & Río de Janeiro & Santos & 210 & 0,6 \\
\hline \multirow[t]{3}{*}{ Tramo 5} & Santos & Montevideo & 901 & 2,0 \\
\hline & & & 15.801 & 35 \\
\hline & Total puertos $=11$ & $\begin{array}{l}\text { Días prom. en } \\
\text { puerto }=1,35\end{array}$ & & 15 \\
\hline \multicolumn{2}{|c|}{ Total Días Round Trip } & & & 50 \\
\hline
\end{tabular}

Cuadro 6 - Línea 3

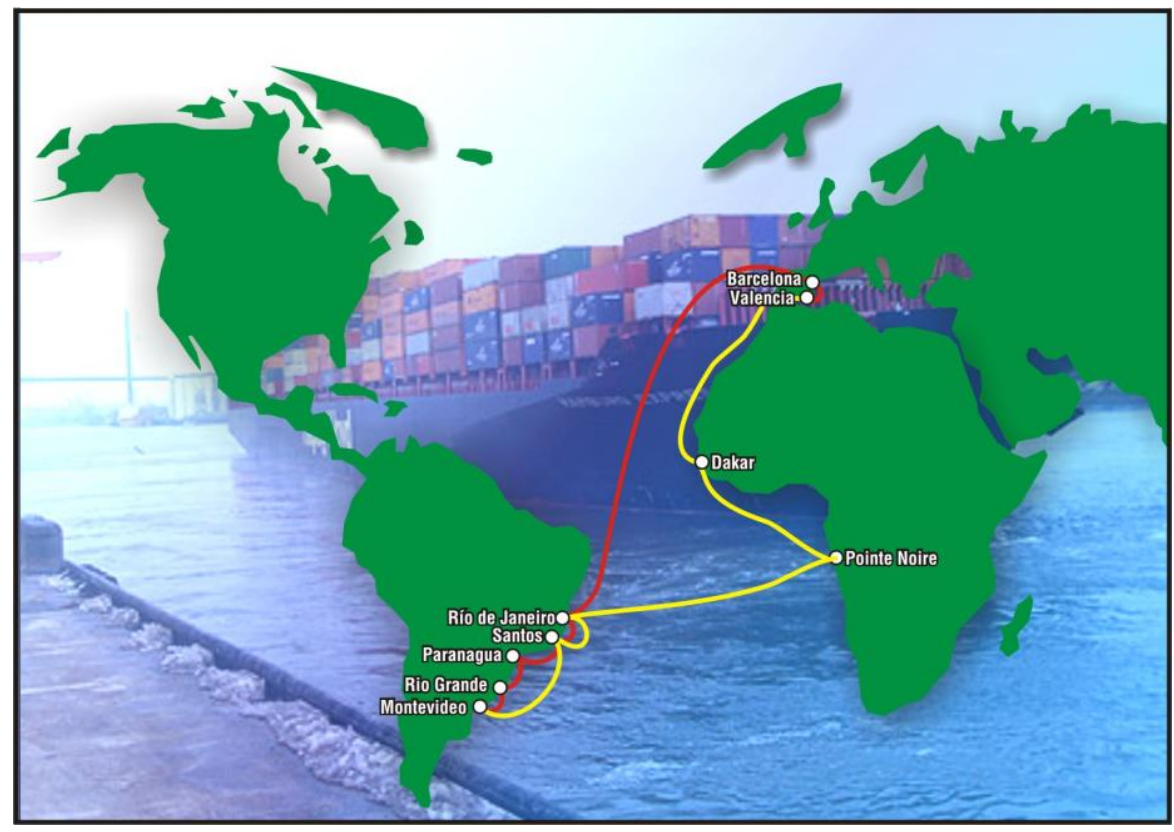

Figura 4 - Ruta Línea 2 
La línea 1 tiene frecuencia semanal, y el round trip tarda 28 días, con lo cual para atender la línea, que recorre un total de 8.204 millas, serán necesarios 4 buques a una velocidad promedio de 19 nudos. Los 4 buques serán Full Container de 2.600 TEU's cada uno, lo cual constituye un supuesto simplificador ya que habitualmente los buques que operan una línea no son exactamente iguales, sino que difieren por ejemplo en capacidad, medida en TEU's, combinando buques de entre 2.600 y 2.800 TEU's.

La línea 2 tiene frecuencia mensual, y el round trip tarda 40 días a una velocidad promedio de 20 nudos, con lo cual para atender la línea, que recorre un total de 13.701 millas, serán necesarios 3 buques. Los 3 buques serán Full Container de 6.500 TEU's cada uno, y serán compartidos con la línea 3, que también tendrá una frecuencia mensual, pero recorrerá 15.801 millas y su round trip será de 50 días a una velocidad promedio de 20 nudos.

En la Tabla 1 se resumen los datos de cada línea y su itinerario:

Tabla 1 - Resumen datos de líneas e itinerarios

\begin{tabular}{|c|c|c|c|}
\hline & Línea 1 & Línea 2 & Línea 3 \\
\hline Cantidad de buques & 4 & 3 & 3 \\
\hline Tamaño del buque (TEU's) & 2.600 & 6.500 & 6.500 \\
\hline Edad (años) & 5 & 5 & 5 \\
\hline Distancia Round Trip (millas) & 8.204 & 13.791 & 15.801 \\
\hline Frecuencia del servicio & semanal & mensual & mensual \\
\hline Días en viaje & 18 & 28 & 35 \\
\hline Días en puerto & 10 & 12 & 15 \\
\hline Días Round Trip & 28 & 40 & 50 \\
\hline Puertos de escala & 7 & 9 & 11 \\
\hline Velocidad & 19 & 20 & 20 \\
\hline
\end{tabular}

Cabe destacar que la edad de los buques que componen la flota, a los efectos de simplificar el presente trabajo, es la misma. Asimismo, se definieron los recorridos de la línea regular de transporte marítimo, suponiendo que ingresarán en todos los puertos de la escala. En la práctica no todos los puertos pueden recibir buques con capacidad mayor a 4.000 TEU's, en particular y a modo de ejemplo se menciona el Puerto de Montevideo, cuya infraestructura y calado, permite el ingreso de buques de hasta 3.500 TEU's. No obstante, es muy probable que en el corto plazo, las Autoridades Portuarias 
de cada país adecuen sus instalaciones para la mejora en la explotación, y por tanto, este supuesto deje de ser tal.

Asimismo, es necesario hacer precisiones en función de la capacidad de la flota de buques y del período de rotación de los contenedores. Es decir, se determina el número de contenedores que como mínimo requiere la empresa para poder operar en función del ratio de rotación que aplique. Para la presente empresa es de 76.700 TEU's. Por otra parte, también se debe decidir sobre el mix de dicha flota de contenedores, o sea sobre la composición por tipo de contenedor y que luego, se adquirirán o arrendarán. Dicho mix será definido sobre la base del tipo de cargas que se comercialicen en cada ruta de navegación, en este caso, la composición es la que sigue:

Tabla 2 - Mix flota contenedores

\begin{tabular}{|c|c|}
\hline Tipo de contenedor & Mix (\%) \\
\hline Común o Dryan (20 pies) & $40 \%$ \\
\hline Común o Dryan (40 pies) & $50 \%$ \\
\hline Reefer (20 pies) & $10 \%$ \\
\hline Total flota & $\mathbf{1 0 0} \%$ \\
\hline
\end{tabular}

En el caso estudiado el $100 \%$ de la flota de contenedores utilizada en todas las líneas es propiedad de la empresa, de modo que no existirán cargos por concepto de arrendamiento o por operativas de leasing comerciales y/o financieros. La casa matriz está establecida en Rotterdam, existiendo 22 filiales (no existen agencias marítimas involucradas) ubicadas en los puertos de escala detallados en el Cuadro 7.

\begin{tabular}{|c|c|c|c|}
\hline Baltimore & Felixstowe & New Orleáns & Puerto Cabello \\
\hline Barcelona & Houston & New York & Río de Janeiro \\
\hline Bremerhaven & Le Havre & Norfolk & Río Grande \\
\hline Cartagena & Miami & Paranagua & Salvador \\
\hline Dakar & Montevideo & Pointe Noire & Santos \\
\hline Vitoria & Valencia & & \\
\hline
\end{tabular}

Cuadro 7 - Filiales de la empresa armadora 
En la Tabla 3 se resumen los resultados comparativos de la aplicación del modelo de costeo descripto para la empresa en términos de costes eficientes determinados a priori- y resultantes directos e indirectos -determinados a posteriori- de la Línea 1:

Tabla 3 - Comparativo costes reales y eficientes Línea 1

\begin{tabular}{|c|c|c|c|c|c|c|}
\hline \multirow[b]{2}{*}{ Factor } & \multicolumn{2}{|c|}{ Coste Eficiente } & \multicolumn{2}{|c|}{ Coste Real sin capacidad ociosa } & \multicolumn{2}{|c|}{$\begin{array}{c}\text { Variación Por } \\
\text { Línea }\end{array}$} \\
\hline & $\begin{array}{l}\text { Por } \\
\text { Round } \\
\text { Trip }\end{array}$ & $\begin{array}{c}\text { Línea } 1 \text { Anual } \\
\text { (52,12 Round } \\
\text { Trip) }\end{array}$ & $\begin{array}{c}\text { Por } \\
\text { Round Trip }\end{array}$ & $\begin{array}{c}\text { Línea } 1 \text { Anual } \\
(52,12 \text { Round Trip) }\end{array}$ & US\$ & $\%$ \\
\hline \multicolumn{7}{|l|}{ Combustible } \\
\hline Maquina principal & 105.056 & 5.475 .519 & 123.435 & 6.433 .432 & \begin{tabular}{|c|}
957.91 \\
3 \\
\end{tabular} & $17,49 \%$ \\
\hline Maquina Auxiliar & 4.286 & 223.386 & 3.983 & 207.594 & \begin{tabular}{|c|}
$(15.79$ \\
$2)$ \\
\end{tabular} & $-7,07 \%$ \\
\hline \multicolumn{7}{|l|}{ Avituallamiento } \\
\hline Repuestos & 6.120 & 318.974 & 5.200 & 271.024 & $\begin{array}{c}(47.95 \\
0)\end{array}$ & $-15,03 \%$ \\
\hline Lubricantes & 6.900 & 359.628 & 6.818 & 355.354 & $(4.274)$ & $-1,19 \%$ \\
\hline Provisiones & 3.400 & 177.208 & 4.247 & 221.354 & 44.146 & $24,91 \%$ \\
\hline Água & 664 & 34.608 & 851 & 44.354 & 9.746 & $28,16 \%$ \\
\hline Tripulación & 33.978 & 1.770 .933 & 32.660 & 1.702 .239 & \begin{tabular}{|c|}
$(68.69$ \\
$4)$
\end{tabular} & $-3,88 \%$ \\
\hline Servicios Portuarios & 212.500 & 11.075 .500 & 202.927 & 10.576 .555 & $\begin{array}{c}(498.9 \\
45)\end{array}$ & $-4,50 \%$ \\
\hline Comunicación & 4.981 & 259.610 & 4.250 & 221.510 & $\begin{array}{c}(38.10 \\
0)\end{array}$ & $-14,68 \%$ \\
\hline Rep. y Mantenimiento & 63.773 & 3.323 .849 & 58.649 & 3.056 .786 & \begin{tabular}{|c|}
$(267.0$ \\
$63)$ \\
\end{tabular} & $-8,03 \%$ \\
\hline Seguros & 47.676 & 2.484 .873 & 42.925 & 2.237 .251 & \begin{tabular}{|c|}
$(247.6$ \\
$22)$ \\
\end{tabular} & $-9,97 \%$ \\
\hline $\begin{array}{c}\text { Bienes de consumo } \\
\text { diferido }\end{array}$ & 160.778 & 8.379 .749 & 160.500 & 8.365 .260 & $\begin{array}{c}\left(\begin{array}{c}14.48 \\
9)\end{array}\right. \\
\end{array}$ & $-0,17 \%$ \\
\hline $\begin{array}{c}\text { Total costes directos } \\
\text { al buque }\end{array}$ & 650.112 & 33.883.837 & 646.445 & 33.692 .713 & \begin{tabular}{|c|}
$(191.1$ \\
$24)$
\end{tabular} & $-0,56 \%$ \\
\hline \multicolumn{7}{|l|}{$\begin{array}{c}\text { Manejo de } \\
\text { contenedores }\end{array}$} \\
\hline Coste por TEU & 6.895 & 359.367 & 6.895 & 359.367 & - & $0,00 \%$ \\
\hline Coste por movimiento & 663.000 & 34.555 .560 & 663.000 & 34.555 .560 & - & $0,00 \%$ \\
\hline Coste refrigeración & 66.300 & 3.455 .556 & 66.300 & 3.455 .556 & - & $0,00 \%$ \\
\hline Coste de intermodalidad & 497.250 & 25.916 .670 & 497.250 & 25.916 .670 & - & $0,00 \%$ \\
\hline $\begin{array}{c}\text { Coste de } \\
\text { reposicionamiento }\end{array}$ & 78.000 & 4.065 .360 & 78.000 & 4.065 .360 & - & $0,00 \%$ \\
\hline $\begin{array}{c}\text { Total costes directos a } \\
\text { la carga }\end{array}$ & 1.311 .445 & 68.352.513 & 1.311.445 & 68.352.513 & - & - \\
\hline
\end{tabular}




\begin{tabular}{|c|c|c|c|c|}
\hline $\begin{array}{c}\text { Costes indirectos } \\
\text { Carga L1 }\end{array}$ & 10.242 .491 & 10.304 .414 & 61.923 & $0,60 \%$ \\
\hline \multicolumn{5}{|l|}{$\begin{array}{c}\text { Costes indirectos } \\
\text { Buques }\end{array}$} \\
\hline $\begin{array}{c}\text { (4 buques de } 2.600 \\
\text { TEU) }\end{array}$ & 16.299.276 & 16.511 .700 & $\begin{array}{c}212.42 \\
4\end{array}$ & $1,30 \%$ \\
\hline Total costes indirectos & 26.541.767 & 26.816.114 & $\begin{array}{c}274.34 \\
7\end{array}$ & $1,03 \%$ \\
\hline $\begin{array}{c}\text { TOTAL COSTES LINEA } \\
1\end{array}$ & 128.778.118 & 128.861.341 & 83.223 & $0,06 \%$ \\
\hline
\end{tabular}

\section{REFLEXIONES FINALES}

El sistema que fue diseñado será aplicado con base a la combinación de los diferentes modelos de costeo puros existentes: completo, variable, resultante y estándar. Dicha combinación será elegida dependiendo de la decisión que se requiera tomar al nivel gerencial, ya que "existen diferentes costes para diferentes decisiones". El modelo definido en el presente trabajo tiene como objeto la adecuada asignación de costes indirectos, por tanto, no debe confundirse con la adecuada toma de decisiones.

En principio, el cálculo de rentabilidades será factible, sin que con la utilización de este modelo se llegue a interrogantes asociadas a - liminación de líneas de servicios, eliminación de escalas y/o recorridos -. Para llegar a tal extremo, recomendamos la utilización del modelo de costeo variable con análisis de costos fijos específicos y programables, ampliamente tratados por Kaplan (2003).

Al efectuar este análisis se observó que estas empresas tienen una gran proporción de costes fijos en su estructura de costes, algunos de los cuales son fijos respecto al viaje y otros son fijos totales al nivel de la empresa, por lo que el inadecuado tratamiento de los mismos, podría derivar en decisiones erróneas. Por ejemplo, el análisis de la posibilidad de sustituir y/o eliminar una línea de servicio bajo un modelo completo puede arribar a conclusiones equivocadas si no se hace un exhaustivo análisis de evitabilidad bajo un modelo de costeo variable.

Asimismo, consideramos que el seguimiento de las variaciones de los costes incurridos con respecto a los costes eficientes de cada línea de la empresa, le brinda a 
ésta, información valiosa acerca de su desempeño, y le permite tomar diversas decisiones.

Como características sobresalientes de la operativa de estas empresas de línea regular, se señalan: el cumplimiento estricto de un cronograma predeterminado; una inversión fija en buques que deben cumplir con las frecuencias pactadas, inflexibilización de la capacidad de manejar la oferta; altos costes de administración por la gran cantidad de personal que requieren, así como también las estacionalidades y los desbalances comerciales.

Por otra parte, cabe mencionar que las tarifas de fletes de línea son reguladas por el mercado, dependiendo del tipo de carga y de la capacidad ociosa prevista de los buques para el período en cuestión, en definitiva son sensibles a las fluctuaciones en el volumen de las cargas en el ámbito mundial.

Todo ello deriva en ingresos elevadamente volátiles para las empresas en cuestión, y dificulta su gerenciamiento. De ello, se desprende que la rentabilidad de una empresa marítima dependerá fuertemente de la eficiencia con la que la misma sea administrada, ya que por el lado de los ingresos no poseen capacidad de maniobra. Para lograr la eficiencia es necesario contar con información adecuada y oportuna para la toma de decisiones, siendo entonces la variable clave a conocer y controlar el coste de su operación.

Consideramos que el presente trabajo intenta abrir una discusión en el sector marítimo y es un granito de arena para la línea empírica que el management accounting ha emprendido en estos últimos años.

\section{REFERÊNCIAS}

AECA (2006). La contabilidad de Gestión en el Sistema Portuario Español, Documento Nro.31 Principios Contabilidad de Gestión. Madrid.

BACIC, M. J. (2006). Modelo para melhoria de processos aplicado na gestao de custos. Revista Universo Contábil 2(3), 9-24. 
BARTLETT, H. C. (1986). Prelude Corporation and Acquaculture. Journal of Management Case Studies 2(4), 346-353.

BREWER, J. \& HUNTER, A. (1989). Multimethod Research: A Synthesis of styles. Newbury Park, C.A.: Sage Publications.

CRESPO TRUJILLO, A., GINER FILLOL, A., PONTET UBAL, N. \& RIPOLL FELIU, V. (2005). La contabilidad de costos de las autoridades portuarias de Valencia y Marín de Pontevedra: Modelo de Mínimos. Boletín Técnico Colegio de Contadores, Economistas y Administradores del Uruguay 29, 55-60.

DE CORLA-SOUZA, P., EVERETT, J., GARDNER, B., \& CULP, M. (1997). Total cost analysis: An alternative to benefit-cost analysis in evaluating transportation alternatives. Transportation 24(2), 107-123.

GINER FILLOL, A. \& RIPOLL FELIU, V. (2007). De la contabilidad de costes a la contabilidad de gestión. El caso de la Autoridad Portuaria de Valencia - Valenciaport. Partida Doble (184), 62-72.

GINER FILLOL, A., PONTET UBAL, N. \& RIPOLL FELIU, V. (2008). Gestión avanzada de la contabilidad de costos en la Autoridad Portuaria de Valencia - Valenciaport (APV). In: Logística e gestao portuaria: uma visao ibero-americana, pp. 175-194. Caxias do Sul: Editorial EDUCS.

. (2007a). Evolución y estrategia de futuro de la gestión de costes: el caso de la Autoridad Portuaria de Valencia. Revista Iberoamericana de Contabilidad de Gestión V(10), 177-198.

. (2007b). Caso práctico: la contabilidad de gestión en el plan estratégico de la Autoridad Portuaria de Valencia. Harvard-Deusto Finanzas y Contabilidad 80(noviembre/diciembre).

HORNGREN, C., FOSTER, G., \& DATAR, S. (1996). Contabilidad de Costes. Un enfoque gerencial. Mexico, D.F.: Prentice Hall.

KAPLAN, A. (2003). EI MOEP y el PEEP, luces amarillas en el tablero de las decisiones empresariales. VIII Congreso del Instituto Internacional de Costos. Punta del Este (noviembre).

LIEB, R. C. \& RANDALL, H. (1999). Use of third-party logistics services by large US manufacturers in 1997 and comparisons with previous years. Transport Reviews 19(2), 103-115.

PUNAKIVI, M. \& HINKKA, V. (2006). Selection Criteria of Transportation Mode: A Case Study in Four Finnish Industry Sectors. Transport Reviews 26(2), 207-219. 
Modelo de Costos Completo para Empresas de Carga Marítima: Caso de Aplicación Norma Pontet Ubal

Data de Submissão: 23/06/2010

Data de Aceite: 20/12/2010 